\title{
Altered Dynamics for USP Apparatus 2
}

Paul G. King, Ph.D.

Paul G. King Consulting Lake Hiawatha, N7

\section{Intraduction}

After reading the article "Improved Hydrodynamics for USP Apparatus 2"(6) I was dismayed that the authors seemed to have confused the concept of "change" with that of "improvement." If only the alteration of the design of the standard dissolution vessel had (a) removed the effect of "deaeration" as they claim without (b) altering the rate of dissolution, then perhaps the alteration would indeed have been an improvement; but neither was shown to be the case.

I agree with part of what their abstract states namely that "USP Apparatus 2 bas been plagued by" problems with "calibrators even when there is nothing wrong with calibrators or the instruments." However, outside of their usually finding higher values when the PEAK vessels were used, the rest of their comments were not supported by the analytical data that they presented. Based on the data presented, I would agree that, in all but one of the cases shown, the new use of the PEAK vessels led to higher dissolution values or accelerated dissolution rates or both at comparable paddle speeds and sampling times. However, given the higher nearer-completion or nearer-limit values reported in the single point studies for the PEAK vessel as compared to the USP vessel, the data did not support:

1. The source of variability has been found to be the hydrodynamics of the vessel;

2. The need for deaeration has been eliminated;

3. The PEAK vessel overcomes the problems caused by the differences in characteristics of currently manufactured brands of vessels; or

4. The PEAK vessel allows for the use of sampling probes of different sizes and characteristics in different locations in the vessels.

Data addressing points " 3 " and "4" was not presented. Moreover, in my experience with Vankel, Distek, Pharma-Test, and Hanson systems, and, where possible, substituting brand with non-brand vessels that met the USP's dimensional specifications and, in some cases switching between brands, the differences in the values observed were less than the variability in the products being tested unless a setup, media, sampling or other such error occurred. The authors did not mention the changes in tablet dynamics and positioning that using the PEAK vessel also produces.

I do agree that "USP Apparatus 2 (paddle method) bas become the workhorse instrument in the modern dissolution laboratory" and that "it is expected to provide rugged and reproducible results, on which decisions about product quality, bioavailability, bioequivalence, etc. are based." However, I must object to the authors' statements:

- "in practice, the apparatus tends to be extremely sensitive to outside and inside variables, with large changes in dissolution profiles resulting from small changes in factors such as paddle rotational speed, vibration, deaeration and insertion of sampling probes $(1,2)$ "

- "dissolution testing is usually regarded as notbing more than a mandated 'service tool' of questionable performance used only to satisfy regulatory requirements, rather than a powerful predictive tool for pharmaceutical research and development (3)."

As an analytical chemist with more than 10 years of experience in dissolution testing, I have found that the chief sources of variability have been, in order of frequency and importance,:

1. The variability in the individual dosage forms being tested,

2. Failure to perform the studies exactly the same way (principally variation in the preparation of the dissolution media or deaeration differences or both),

3. Improper setup of the system (shaft posi- 
tioning, shaft centering, bent shafts, vessels left uncovered, bath level low, etc.) and

4. Controllable instrument factors (vibration, temperature inhomogeneity in the bath, speed errors and fluctuations, improper positioning of the heater discharge in "in the bath" designs, etc.).

With respect to utility, in addition to being a good indicator of dosage-form performance for the setting of expiration dates from stability test data in the development of new drugs, dissolution testing is a key to determining the equivalence of dosage forms made by different manufacturers or processes. It is critical to establishing that manufacturing process changes, excipient changes and other minor formulation changes have not changed the delivery of the active ingredient. The view that dissolution testing is "notbing more than a mandated 'service tool" ignores the fact that the acceptance of dissolution testing by regulatory agencies in the United States and abroad was based on dissolution testing's proven ability to be a surrogate for dosage-form delivery performance. Lacking this tool, pharmaceutical manufacturers would need to do some form of bio-equivalence testing to establish (a) that dosage-form changes do not affect the delivery performance of the finished dosage form or (b) packaging, storage, and time studies needed to set valid expiration dating.

\section{Dynamics}

As recent dissolution studies by Wang and Coffin (4) have shown, the rotation speed of $50 \mathrm{rpm}$ for tablet dissolution can create cones of materials on the bottom of the vessel. These "cones" are the result of the fluid flow present in USP cylindrical, hemispherically bottomed vessels. In cases where tablet disintegration is the key variable controlling dissolution, the build up of the disintegrated particles around the central tablet core does shield the bulk core from the bulk flow of the medium being used in a manner that can retard the dissolution of the active ingredient or ingredients.

The new PEAK (5) vessel does eliminate the possibility of a central cone. The new PEAK vessel has a cone molded into the bottom that causes disintegrating materials to be dispersed into a ring around the molded in cone. In effect, this "molded in cone" displaces the center and causes "disintegrating particles" to be dispersed into a ring around the "cone" which, for a given mass of disintegrated material is larger in area and, of necessity, thinner than the cone of similar material in the USP vessel. In addition, because of the turbulence generated, the tablets tumble around the "molded in cone" much more vigorously than they do in the center of the USP vessel. Finally, positioning the shafts in the PEAK vessels at the distance from the bottom of the molded in peak to meet the USP requirement for placement decreases the effective average distance between the bottom of the active region of the paddle to the top of the dosage unit being tested because they are displaced towards the tips of the paddle blade and cannot reside in the center, the region of least turbulence. 


\section{Altered Dynamics....cont.}

Given all of the preceding differences, one would predict that the PEAK vessel could give higher values than the USP vessel because of the obviously stronger energy transfer mechanisms for the PEAK vessel. Thus, "the glass cone effectively displaces the unstirred cone, forcing the material tested into" a region of different dynamics, where all surfaces of the product are more exposed to the moving medium and to interactions with the tumbling tablet.

Therefore, the PEAK vessel's shape changes not only the hydrodynamics of the vessel but also the dynamics of the tablet motion, the average proximity of the tablet to the paddle, and, for disintegrating tablets, the dynamics of the interaction of the bulk of the tablet with the disintegrated portions of the tablet. The commentary which follows will attempt to evaluate the data presented in the article "Improved Hydrodynamics for USP Apparatus 2" (6).

\section{Influence of Deaeration}

In "Figure 3," the article reports data for studies using two test conditions, "D" and "ND," in both labs using a non-USP "standard" tablet, "FDA Prednisone NCDA\#2 $10 \mathrm{mg}$ Tablets" (a disintegrating tablet), $500 \mathrm{~mL}$ of "water" and $50 \mathrm{rpm}$ with sampling at 30 minutes. The two test conditions correspond to using "deaerated water" ("D") or to using "nondeaerated water" ("ND") as the dissolution media. However, the article does not state the sources of the water used, its quality, the procedure used by each lab to "deaerate" the water, or the measures of the quality of the water. In "Figure 3," the first fact seems to be that when using the PEAK vessels both labs obtained results "similar" to the results obtained by "Lab 2" in the "ND" case. When using the USP vessels and the PEAK vessels, both labs obtained lower average results using "deaerated water." Because all of the data for the PEAK vessel cases was close to the point that the available drug has completely dissolved (> $80 \%$ "released"), the differences between the " $\mathrm{D}$ " cases and the "ND" cases are small. Because the data for the "D" cases using the USP vessels were at values indicating that less than $45 \%$ of the available drug had dissolved and the "ND" values were in the " $50+$ to $90 \%$ " range, the differences seen were much larger when the USP vessels were used. Nonetheless, the "D" values were less than the "ND" values for both the PEAK and the USP vessels. Before any valid conclusion could be drawn as to whether or not the PEAK vessel significantly reduces the "deaeration" effect, the sampling time on the PEAK vessels would need to have been reduced until the "\% dissolved" values were less than $50 \%$ for the "D" cases. In the absence of such data and without replication of at least the "D" experiment in each lab, the data do not support the footnoted statement that the deaeration has "negligible influence on the Peak vessels."

In "Figure 4," the article reports data for studies using the same two test conditions, "D" and "ND," in the same two labs and the USP disintegrating calibrator tablet, "USP Prednisone Calibrator $50 \mathrm{mg}$ Tablets", using $900 \mathrm{~mL}$ of water, $50 \mathrm{rpm}$, and sampling at 30 minutes. The two test conditions correspond to using "deaerated water" ("D") or to using "nondeaerated water" ("ND") as the dissolution media. Based on the data presented in "Figure 4," the use of the PEAK vessels did not produce results that indicate any acceleration or increase of dissolution over the USP vessel. Yet the article does not address or explain why this was the case. Moreover, there appears to be a small "deaeration" effect for the USP vessels in both labs and "Lab 2" for the PEAK vessels; but no apparent effect in "Lab 1" using the PEAK vessels. Given that about 50 to $60 \%$ of the $50 \mathrm{mg}$ of drug was measured under this test condition in $900 \mathrm{~mL}$ of water and that there was not a significantly larger value for experiments using the PEAK vessel, then about $15 \mathrm{mg}$ of Prednisone could have dissolved if the volume were reduced to $500 \mathrm{~mL}$. This confirms that the values above $80 \%$ in the "Figure 3" experiments probably represented almost complete solubilization of the available Prednisone in the PEAK vessels at 50 rpm after 30 minutes.

In "Figure 5," the article reports data for studies using the same two test conditions, "D" and "ND" 
in the same two labs using the USP non-disintegrating Salicylic Acid $300 \mathrm{mg}$ Calibrator tablets 900 $\mathrm{mL}$ of $0.05 \mathrm{M}$ phosphate buffer at $\mathrm{pH} 7.4$, and $50 \mathrm{rpm}$ with sampling at 30 minutes. The two test conditions correspond to using "deaerated buffer" ("D") or to using "nondeaerated buffer" ("ND") as the dissolution media. Based on the data presented in "Figure 5," without any replication data, all that should be said is that, in this case, the use of the PEAK vessels did produce results that indicated an acceleration or increase of dissolution over the USP vessel. In addition, there did not appear to be a significant " $D$ " effect for either the PEAK vessels in both labs and "Lab 2 " for the USP vessel [there is no data "ND" for "Lab 1" in this figure].

Summarizing the article's data on the influence of deaeration, it seems that using the PEAK vessels produces higher values for both disintegrating and non-disintegrating tablets provided the solubility of the drug is not near the solution's capacity. Claims as to the lack of effect of "deaeration" have not been substantiated. Given the higher values, the authors should have reduced the time variable until the mean values observed for the PEAK vessels " $D$ " experiments "matched" those of the USP vessels and then reported the "D" and "ND" values at that time and compared those to the "D" and "ND" values at 30 minutes in the USP vessels.

\section{Influence of Stirring Speed}

In "Figure 6," the article reports data for studies using three rpm values, 50, 75, and 100, in "Lab 1 " and a non-USP "standard" tablet, "FDA Prednisone NCDA\#2 10 mg Tablets” (a disintegrating tablet), using $500 \mathrm{~mL}$ of "deaerated water," and sampling at 30 minutes. Given (Figure 3) that the previous data show that the $10-\mathrm{mg}$ Prednisone tablets are "completely" dissolved at $50 \mathrm{rpm}$ in the PEAK vessels, then increasing the stirring speed should, as it does, have no effect. When a tablet is "completely" dissolved under a fixed time/media/speed condition, then increasing the speed will not affect the amount dissolved and the variations will be those arising from product inhomogeneity and measuring fluctuations. Therefore, as designed, the results of the speed study using the PEAK vessel should not, and did not show any "stirring speed" effect. Based on the data for the USP vessels, it appears that as one increases the speed significantly, the dissolution increases significantly also. For this study to address the issue raised in the article of "large changes in dissolution profiles resulting from small changes in paddle rotational speed," then it would have studied profiles at times where the tablet is not dissolved completely with small perturbations in rpm (for example, 48, 50, $52 \mathrm{rpm}$ ), randomization, triplicate or more experiments at each speed, recovery controls, formal experimental designs, and statistical evaluation to ascertain the difference in results, validly attributable to the speed of rotation.

In "Figure 7," the article reports data for studies using three rpm values, 50, 75, and 100, in "Lab 1" using the USP non-disintegrating calibrator tablet, "USP 


\section{Altered Dynamics....cont.}

Salicylic Acid Calibrator $300 \mathrm{mg}$ Tablets" and 900 $\mathrm{mL}$ of $0.05 \mathrm{M}$ phosphate buffer at $\mathrm{pH} 7.4$ with sampling at 30 minutes. In this case, the PEAK vessels gave higher results at every speed than the USP vessels. The data from the PEAK vessels also appears to reach a maximum at $75 \mathrm{rpm}$. Though lower, the apparent "average" values for the USP test seem to be the "same" at all rpm levels (but only 4 values appear to have been reported at $75 \mathrm{rpm})$. In neither case is there a large "rpm" effect. The only fact that is clearly supported is that the PEAK vessels give higher values than the USP for a 30-minute sampling time or, in the authors' words, "data also indicates that dissolution is more complete in PEAK vessels compared to the conventional cylindrical type."

Summarizing the article's data on the influence of the stirring speed, use of PEAK vessels does indeed produce higher values for both disintegrating and non-disintegrating tablets provided the solubility of the drug is not near the solution's capacity.

\section{Excellent Correlation to Dissolution Results Using USP Apparatus 3}

The article's data as presented in Figures 8,9 \& 10 did show that the reported dissolution results obtained from PEAK vessels using Apparatus 2 correlated closely with those from Apparatus 3. However, the failure: (a) to use a "spiral wire sinker" for all of the USP 2 tests, (b) to make certain that the $\mathrm{pH}$ of the media was 1.0 when the tests were done using the PEAK vessels, (c) to address the problem of much lower results obtained for the PEAK vessels (P1) when the tablets were allowed to be stuck in one position for 6 to 8 hours, and (d) to define the time and method for loosening the tablets in "P2" and "P3" experiments precludes any other valid conclusions from being reached.

However, are higher rates and good correlation between USP 3 and USP 2 desirable? If so, then why not simply move the paddle closer to the bottom?

\section{No Influence of Sampling Probes}

In the absence of any data addressing the influence of sampling probes with the PEAK vessel, the authors' claim of "no influence of sampling points" is not justified.

\section{PEAK Vessels and USP Calibrators}

The authors propose changing the USP's set of specifications, calibrators, and vessels to an as yet undefined PEAK set as the solution to the dissolution differences between results obtained using PEAK vessels and those obtained using USP vessels. However, the concomitant problems of changing the limits, times, or rpm, or combinations thereof for all of the existing products and the dislocations such a change as this would introduce are ignored. Based on a consideration of all that would need to be done to change from the USP vessels to the PEAK vessels, it seems that more problems would be generated than this change purports to solve.

\section{Conclusions}

The use of the PEAK vessels alters the dynamics of the present USP 2 dissolution system. Other than an increase in the values observed at a given point in time below the component saturation point, none of the claims made by the authors for the PEAK vessel in comparison to the USP vessel have been substantiated by the data they have presented.

\section{References}

(1) Moore T. W., Hamilton J. F., and Kerner C. M., Pharmacopeial Forum 1995; 1(5):1387-1396

(2) Wells C. E., Journal of Pharmaceutical Science 1982; 71(6): 232

(3) Beckett H. A., "In-Vitro Dissolution Testing Technology," February 1996 Presentation at Vankel Industries Dissolution Communication and Exhibition, Edison, NJ, USA 


\section{Altered Dynamics... continued}

(4) Wang T. and Coffin D. M., Glaxo Research Institute 1995 AAPS Poster No. APQ 1134

(5) PEAK Vessel ${ }^{\text {tw }}$, patent-pending from VanKel Industries, Inc.

(6) Beckett A. H., Quach T.T., and Kurs G.S., Dissolution Technologies 1996; 3(2): 7-10 and 18. 